\title{
Changes and prognostic impact of inflammatory nutritional factors during neoadjuvant chemoradiotherapy for patients with resectable and borderline resectable pancreatic cancer
}

\author{
Minoru Oshima ${ }^{1 *} \mathbb{0}$, Keiichi Okano ${ }^{1}$, Hironobu Suto ${ }^{1}$, Yasuhisa Ando ${ }^{1}$, Hideki Kamada², Tsutomu Masaki²,
} Shigeo Takahashi ${ }^{3}$, Toru Shibata ${ }^{3}$ and Yasuyuki Suzuki ${ }^{1}$

\begin{abstract}
Background: Inflammatory nutritional factors, such as the neutrophil/lymphocyte ratio (NLR), Glasgow Prognostic Score (GPS), modified GPS (mGPS), and C-reactive protein/albumin (CRP/Alb) ratio, have prognostic values in many types of cancer. In this study, the prognostic values of inflammatory nutritional scores were evaluated in the patients with resectable or borderline resectable pancreatic ductal adenocarcinoma (PDAC) after neoadjuvant chemoradiotherapy (NACRT).

Methods: A total of 49 patients who underwent pancreatectomy after NACRT from September 2009 to May 2016 were enrolled. The NACRT consisted of hypofractionated external-beam radiotherapy (30 Gy in 10 fractions) with concurrent S-1 $\left(60 \mathrm{mg} / \mathrm{m}^{2}\right.$ ) delivered 5 days/week for 2 weeks before pancreatectomy. Inflammatory nutritional scores were determined before and after NACRT in this series.

Results: The median NLR increased after NACRT (from 2.067 to 3.302), with statistical difference $(p<0.001)$. In multivariate analysis, high pre-NACRT mGPS (2 or $1 ; p=0.0478$ ) and significant increase in CRP/Alb ratio after NACRT $(\geqq 0.077 ; p=0.0036)$ were associated with shorter overall survival. All patients were divided into two groups according to the $\triangle C R P / A l b$ ratio after NACRT: the group with high $\triangle C R P / A l b$ ratio $(\geqq 0.077)$ and the group with low $\triangle C R P / A l b$ ratio (<0.077). The group with high $\triangle C R P / A l b$ ratio after NACRT $(n=13)$ not only had higher post-NACRT CRP levels $(p<0.001)$ but also had lower post-NACRT Alb levels $(p=0.002)$. Patients in the group with high $\triangle C R P / A l b$ ratio lost more body weight during NACRT ( $p=0.03)$.
\end{abstract}

Conclusion: In addition to pre-NACRT mGPS, $\triangle$ CRP/Alb after NACRT could provide prognostic value in the patients with PDAC treated by NACRT.

Keywords: Inflammatory nutritional factor, PDAC, NACRT

*Correspondence: m-oshima@med.kagawa-u.ac.jp

${ }^{1}$ Department of Gastroenterological Surgery, Faculty of Medicine, Kagawa University, 1750-1, Ikenobe, Miki-cho, Kita-gun, Kagawa 761-0793, Japan

Full list of author information is available at the end of the article

\section{Background}

Pancreatic ductal adenocarcinoma (PDAC), as a representative lethal malignant disease, is the fourth leading cause of cancer death worldwide and has the worst prognosis, with less than $8 \%$ of patients surviving 5 years

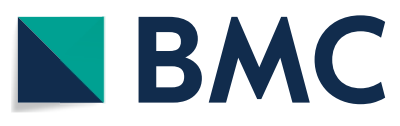

(c) The Author(s) 2020. Open Access This article is licensed under a Creative Commons Attribution 4.0 International License, which permits use, sharing, adaptation, distribution and reproduction in any medium or format, as long as you give appropriate credit to the original author(s) and the source, provide a link to the Creative Commons licence, and indicate if changes were made. The images or other third party material in this article are included in the article's Creative Commons licence, unless indicated otherwise in a credit line to the material. If material is not included in the article's Creative Commons licence and your intended use is not permitted by statutory regulation or exceeds the permitted use, you will need to obtain permission directly from the copyright holder. To view a copy of this licence, visit http://creativecommons.org/licenses/by/4.0/. The Creative Commons Public Domain Dedication waiver (http://creativeco mmons.org/publicdomain/zero/1.0/) applies to the data made available in this article, unless otherwise stated in a credit line to the data. 
after diagnosis [1]. Although surgical resection provides only chance for cure, the resection rate is very low (15$20 \%$ ) [2]. Additionally, even after surgical resection with curative pathological findings, an estimated $80-85 \%$ of patients experience recurrence, with a median survival of 20-24 months [3-5]. The therapeutic agent added to surgery, particularly adjuvant chemotherapy, appears to be necessary and effective $[2,6]$. Advanced research in adjuvant and neoadjuvant therapies, including chemotherapy, radiotherapy, and chemoradiotherapy, has focused on its therapeutic effect to PDAC [7-10].

Determining the prognostic factors is of importance in facilitating adequate treatment for patients with PDAC and identifying patients who might have poor prognosis and would not benefit from the treatment. Some studies have described various prognostic predicting factors for patients with resectable (R) or borderline resectable (BR) PDAC, e.g., age, tumor size, tumor marker carbohydrate antigen 19-9 level, resection margins, lymph node metastasis, and postoperative complications [11-14].

Recently, inflammation-based prognostic scores and nutritional status, such as the neutrophil/lymphocyte ratio (NLR), C-reactive protein/albumin (CRP [mg/L]/ Alb [g/L]), Glasgow Prognostic Score (GPS), and modified GPS (mGPS), are considered to be associated with tumor progression and prognosis in various carcinomas [15-19]. Some research indicated that inflammatory nutritional scores are associated with the prognosis of the patients with PDAC $[15,20,21]$. However, few reports have described the values of such prognostic scores focusing on the patients treated by neoadjuvant chemoradiotherapy (NACRT), including those with other types of cancers [22].

NACRT was introduced for R and BR-PDAC in 2009 with approval from the ethical committee in our institution [23]. In the current study, the prognostic values of inflammatory nutritional scores were evaluated in the patients who had been enrolled in this Phase II clinical trial.

\section{Methods \\ NACRT}

The previous NACRT study was a single-center, prospective phase II trial in the patients who had histologically diagnosed PDAC (UMIN-CTR \#UMIN000026438). Hypofractionated external-beam radiation (30 Gy in 10 fractions) with concurrent S-1 (60 mg/m2/day) was delivered 5 days per week for 2 weeks. After restaging, pancreatic resection was performed appropriately 4 weeks after the initiation of the protocol treatment. Eligibility criteria of this NACRT protocol included patients with R and BR PDAC (based on the NCCN guideline) [24], performance status $0-1$, age $20-85$ years, adequate organ function (defined by hemoglobin $\geq 9 \mathrm{~g} / \mathrm{dL}$, absolute neutrophil count $\geq 2 \times 10^{9} / \mathrm{L}$, platelet count $\geq 100 \times 10^{9} / \mathrm{L}$, total bilirubin $\leq 6.0 \mathrm{mg} / \mathrm{dL}$, serum transaminases $\leq 3$ times the upper normal limit, and creatinine clearance $\geq 60 \mathrm{~mL} /$ min [23]. All patients provided written informed consent before inclusion in the study, which was approved by the institutional review board of Kagawa University. This phase II trial enrolled 57 patients from September 2009 to May 2016.

\section{Patient selection}

Fifty-five patients eventually underwent pancreatic resection after the NACRT. Excluding 6 patients who were complicated with infection diseases, mostly acute cholangitis, and had autoimmune diseases required medication before or during NACRT, a total of 49 patients were included the present study (Fig. 1).

\section{Surgery}

Pancreaticoduodenectomy (PD) with regional lymphadenectomy was performed according to the Whipple procedure. The reconstruction after PD was performed with the modified Child's method. Distal pancreatectomy (DP)

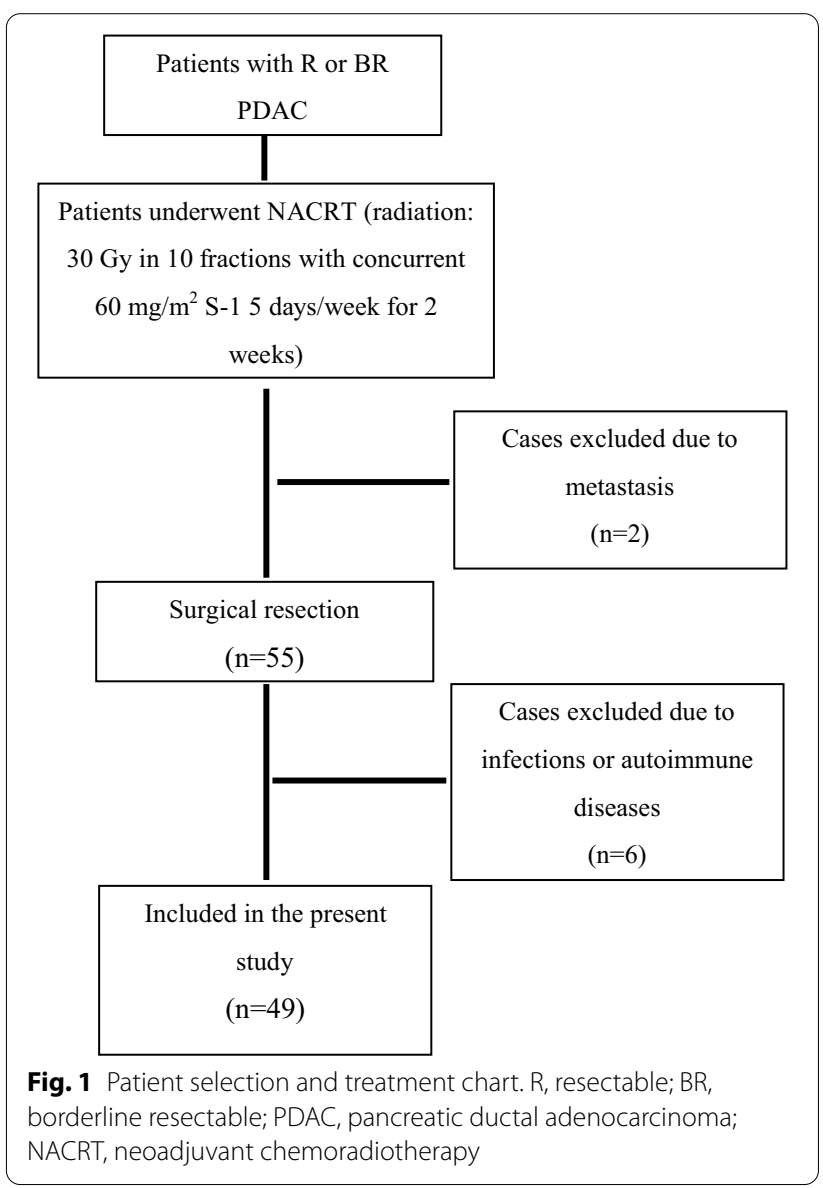


was performed basically with radical antegrade modular pancreatosplenectomy (RAMPS) procedures.

\section{Definition of inflammatory nutritional prognostic scores}

The inflammatory nutritional prognostic scores were determined before NACRT and after NACRT (before surgical resection) in this study. CRP/Alb meant CRP (mg/L)-albumin (g/L) ratio. GPS and mGPS were calculated in accordance with original papers as descried in Additional file 1: Table S1 [18, 19].

\section{Statistical analyses}

The survival curve was estimated using the KaplanMeier method. Overall survival (OS) and disease-free survival (DFS) were defined as the interval from the start of NACRT to death from any cause and recurrence or to the last follow-up. Statistical data were obtained using the BellCurve for Excel (Social Survey Research Information Co., Ltd.). The $75 \%$ level of the continuous variables was defined as the cutoff for the statistical analyses.

\section{Comparison based on inflammatory nutritional scores}

Considering the results of the prognostic scores, the patients in the present study were divided into two groups based on the inflammatory nutritional scores. Additional assessment was performed to determine the factors affecting the inflammatory nutritional scores associated with poor PDAC prognosis.

\section{Results}

Table 1 summarized the patient characteristics. The tumor was located in the proximal pancreas in 36 patients and in the distal pancreas in 13.Forty-one patients with R PDAC (84\%) and 8 with BR PDAC (16\%) were included. Seven patients had BR with involvement of the portal vein (BR-PV) and 1 had BR with arterial abutment (BR-A). R0 resection was achieved in 35 of the 49 patients $(71.4 \%)$. Pathological tumor response was classified as Evans grade I in 3 patients, IIa in 30 patients, IIb in 14 patients, III in 1 patient, and IV in 1 patient. The postoperative morbidity (Clavien-Dindo grade IIIb or more) was observed in $12(25 \%)$ patients. Twenty-seven (55\%) patients completed adjuvant chemotherapy with S-1 or gemcitabine for 6 months according to the recommended protocols. The median observation period of this study was 33 months. In the present series, the 1-, 3-, and 5-year OS rates with the Kaplan-Meier survival estimates were $89.6 \%, 52.5 \%$, and $39.4 \%$, respectively.

Changes of inflammatory nutritional scores before NACRT (pre NACRT), after NACRT (post NACRT) (equal to immediately before surgery) and 3 months after surgery are shown in Fig. 2. NLR increased after NACRT (from 2.067 to 3.302) and decreased 3 months
Table 1 Patient characteristics $(n=49)$

\begin{tabular}{lc}
\hline Age (years) & $70(61-82)$ \\
Tumor size $(\mathrm{mm})$ & $22(10-70)$ \\
Sex & $27(55.1)$ \\
Male & $22(44.9)$ \\
Female & \\
Primary tumor location & $36(73.5)$ \\
Head/neck & $13(26.5)$ \\
Body/tail & \\
Operation procedure & $34(69.4)$ \\
Pancreatoduodenectomy & $11(22.4)$ \\
Distal Pancreatectomy & $4(8.2)$ \\
Total Pancreatectomy & \\
NCCN resectability (2015) & $41(83.7)$ \\
Resectable & \\
Borderline resectable & $7(14.3)$ \\
$\quad$ BR-PV & \\
BR-A & \\
Operation time (min) & $1(2)$ \\
Intraoperative bleeding (mL) & $477(231-816)$ \\
Postoperative hospital stays (days) & $1360(231-9268)$ \\
Complication after surgery & $25(14-152)$ \\
Uncompleted adjuvant chemotherapy & $12(24.5)$ \\
\hline Data are presentedas medan (range) & $22(44.9)$ \\
\hline
\end{tabular}

Data are presented as median (range) or $\mathrm{n}(\%)$. Each value was assessed before neoadjuvant chemoradiotherapy

NCCN, National Comprehensive Cancer Network; BR-PV, borderline resectable with portal vein invasion; BR-A, borderline resectable with arterial invasion

a According to NCCN guideline 2018 [1]

after surgery (from 3.302 to $2.148, p=0.03$ ) with statistically significant differences $(p<0.001$ and $p=0.03$, respectively) (Fig. 2a). In contrast, GPS, mGPS and CRP/ Alb did not change significantly between NACRT and at 3 months after surgery (Fig. $2 b-d$ ).

According to the statistical analysis, the $75 \%$ level of the continuous variable was defined as the cutoff (Table 2). In the univariate Cox regression analysis, resectability (borderline resectable; $p=0.003$ ), high pre-NACRT mGPS ( 2 or $1 ; p=0.007$ ), high post-NACRT CRP/Alb ratio $(\geqq 0.241 ; p=0.0012)$, and significant increase in CRP/Alb ratio (high $\triangle \mathrm{CRP} / \mathrm{Alb}$ ) after NACRT ( $\geqq 0.077 ; p=0.006$ ) were significantly associated with shorter OS. Tumor size in post-NACRT was not included in the regression analysis because it is difficult to measure tumor size accurately due to effect of radiation.

According to the perioperative course and pathological findings, intraoperative bleeding ( $\geqq 2088 \mathrm{~mL} ; p=0.04)$, lymph node metastasis (N1 or N2; $p=0.008$ ), portal vein invasion (PVI; 0.0001), and positive surgical margin (R1; $p=0.004)$ were significantly associated with shorter OS.

In the multivariate survival analysis using a Cox proportional hazards model, high pre-NACRT mGPS ( 2 or 


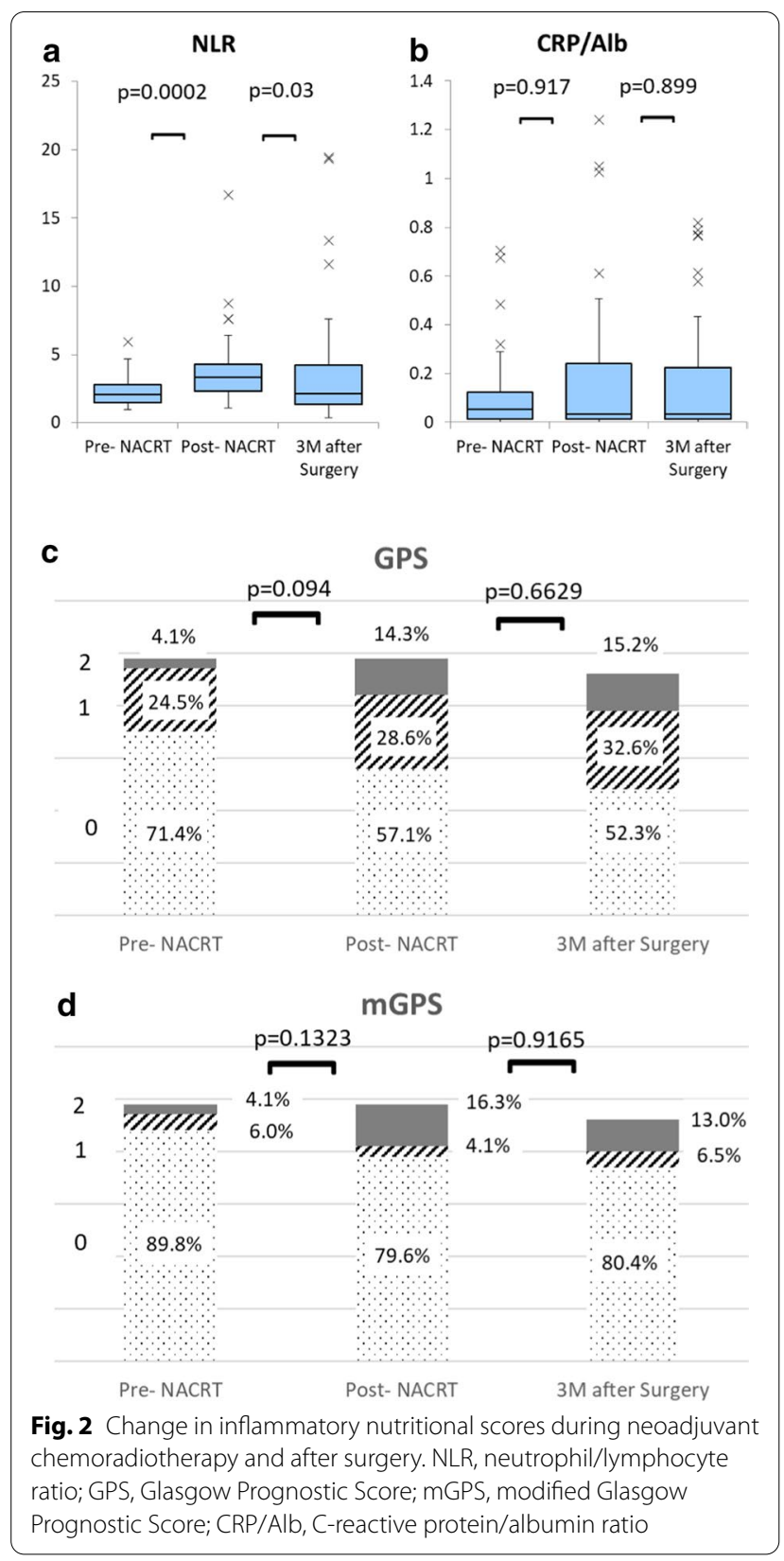

$1 ; p=0.0478$ ), significant increase in CRP/Alb ratio (high $\triangle \mathrm{CRP} / \mathrm{Alb})$ after NACRT $(\geqq 0.077 ; p=0.0036)$, lymph node metastasis ( $\mathrm{N} 1$ or $\mathrm{N} 2 ; p=0.0117)$, and positive surgical margin ( $1 ; p=0.0295)$ were associated with shorter OS, with statistical differences (Table 2). The prognostic value of high post-NACRT CRP/Alb ratio was excluded in the multivariate analysis because high postNACRT CRP/Alb ratio was closely related with a significant increase in CRP/Alb ratio after NACRT.

The Kaplan-Meier survival estimates showed poor OS, with median OS of 12.8 months in the group with high pre-NACRT mGPS (2 or 1 ) compared with 59.3 months in the group with low pre-NACRTmGPS (0) $(p=0.003)$ (Fig. 3a). In addition, median OS were 23.1 and 64.1 months in the high $(\geqq 0.077)$ and low $(<0.077)$ $\triangle \mathrm{CRP} /$ Alb groups $(p=0.004)$ (Fig. $3 \mathrm{~b})$. The N2 or N1 group also demonstrated poor OS, with statistical differences $(p=0.006)$ (Fig. 3c).

DFS were analyzed with the Kaplan-Meier log-rank test. DFS in the group with high pre-NACRT mGPS (2 or 1) and the N1 group were poor, with statistical differences ( $p=0.016, p=0.0002$ ) (Fig. $4 \mathrm{a}, \mathrm{c}$ ). Although the $\mathrm{p}$-value did not reach statistical difference, DFS of the group with high $\triangle \mathrm{CRP} / \mathrm{Alb}$ was shorter compared with the group with low $\Delta \mathrm{CRP} / \mathrm{Alb}(p=0.0573)$ (Fig. $4 \mathrm{~b})$.

All the patients included this study were divided into two groups according to $\triangle \mathrm{CRP} / \mathrm{Alb}$ ratio after NACRT: the group with high $\triangle \mathrm{CRP} / \mathrm{Alb}$ ratio $(\geqq 0.077)$ and the group with low $\triangle$ CRP/Alb ratio $(<0.077)$ (Table 3). The group with high $\triangle$ CRP/Alb ratio after NACRT $(n=13)$ not only had higher post-NACRT CRP levels $(p<0.001)$ but also had lower post-NACRT Alb levels $(p=0.0015)$ than the group with low $\triangle \mathrm{CRP} / \mathrm{Alb}$ ratio. The patients with high $\triangle \mathrm{CRP} / \mathrm{Alb}$ ratio lost more body weight after NACRT $(p=0.03)$. There was no significant difference in tumor-related factors (e.g., tumor size, tumor marker, or lymph node metastasis) between the high and low $\triangle \mathrm{CRP} /$ Alb groups.

\section{Discussion}

The prognosis of PDAC depends on biological behavior and patient-related factors $[2,25]$. Some factors are known predictive prognosis factors of PDAC, such as age, tumor size, pathological type, lymph node metastasis, and vascular invasion [2]. Recently, some papers described patient-related factors that affect the prognosis of some cancers that are as useful as common prognosis factors. Inflammation- and nutrition-based prognostic scores, such as the NLR, GPS, mGPS, and CRP/Alb ratio have been reported to have prognostic values in many types of cancer [15-19]. Chronic inflammation has a direct causal relationship with tumorigenesis, and malignancies trigger an inflammatory response that leads to deleterious effects on the malignant process [26]. Cancerrelated inflammation causes suppression of antitumor immunity through recruitment of regulatory $\mathrm{T}$ cells and activation of chemokines that encourage tumor growth and metastasis [27]. Patients with PDAC are usually malnourished and thus immunocompromised [28]. Impaired host immunity can contribute to tumor growth [29]. Some studies of inflammatory response-related scoring systems in assessing the prognosis of patients with PDAC concluded that NLR, GPS, mGPS, and CRP/Alb ratio may be useful for predicting prognosis $[15,20,21]$. 
Table 2 Analysis of the association between clinical characteristics and OS

\begin{tabular}{|c|c|c|c|c|c|}
\hline & \multirow[t]{2}{*}{ Cutoff value } & \multicolumn{2}{|c|}{ Univariate analysis } & \multicolumn{2}{|c|}{ Multivariate analysis } \\
\hline & & Hazard ratio & $p$ value & Hazard ratio & $p$ value \\
\hline Age (years) & $\geqq 70$ & 1.1455 & 0.7395 & & \\
\hline Sex & Female & 1.4304 & 0.3828 & & \\
\hline Location & Head & 0.8938 & 0.8142 & & \\
\hline Resectability & BR-A or BR-PV & 3.9383 & 0.0033 & 0.9127 & 0.8896 \\
\hline \multicolumn{6}{|l|}{$<$ Pre-NACRT $>$} \\
\hline Tumor size (mm) & $\geqq 20$ & 1.6170 & 0.3436 & & \\
\hline CEA (ng/mL) & $\geqq 5.8$ & 0.9582 & 0.9289 & & \\
\hline CA19-9 (U/mL) & $\geqq 993$ & 1.6661 & 0.2444 & & \\
\hline FDG-PET (SUVmax) & $\geqq 10.87$ & 0.9028 & 0.8294 & & \\
\hline NLR & $\geqq 2.79$ & 2.2490 & 0.0583 & & \\
\hline Alb (g/dL) & $\leqq 3.5$ & 0.9777 & 0.9624 & & \\
\hline GPS & 2 or 1 & 1.3676 & 0.4946 & & \\
\hline mGPS & 2 or 1 & 4.7085 & 0.007 & 3.8635 & 0.0478 \\
\hline CRP/Alb ratio & $\geqq 0.124$ & 2.2201 & 0.0738 & & \\
\hline \multicolumn{6}{|l|}{$<$ Post-NACRT $>$} \\
\hline CEA (ng/mL) & $\geqq 6.5$ & 1.0509 & 0.1986 & & \\
\hline CA19-9 (U/mL) & $\geqq 483$ & 1.1494 & 0.7716 & & \\
\hline NLR & $\geqq 4.30$ & 1.9694 & 0.1229 & & \\
\hline Alb (g/dL) & $\leqq 3.2$ & 2.1969 & 0.0665 & & \\
\hline GPS & 2 or 1 & 1.9590 & 0.1087 & & \\
\hline mGPS & 2 or 1 & 2.3647 & 0.0603 & & \\
\hline CRP/Alb ratio & $\geqq 0.24$ & 2.9130 & 0.00122 & & \\
\hline \multicolumn{6}{|c|}{$<$ Change of inflammatory response factor after NACRT $>$} \\
\hline \multicolumn{6}{|l|}{$\triangle:$ Post-NACRT-pre-NACRT } \\
\hline$\triangle N L R$ & 1.79 & 0.9952 & 0.9971 & & \\
\hline GPS & Increase & 2.2562 & 0.0583 & & \\
\hline mGPS & Increase & 2.3647 & 0.0603 & & \\
\hline$\triangle \mathrm{CRP} /$ Alb ratio & 0.0769 & 3.2706 & 0.0060 & 5.1842 & 0.0036 \\
\hline \multicolumn{6}{|l|}{$<$ Perioperative factor $>$} \\
\hline Operative time (min) & $\geqq 560$ & 1.8544 & 0.1999 & & \\
\hline Intraoperative bleeding (mL) & $\geqq 2088$ & 2.4717 & 0.0387 & 1.1879 & 0.7548 \\
\hline Complication after surgery & $\geqq I l l a$ & 0.6719 & 0.4338 & & \\
\hline Postoperative hospital stays (days) & $\geqq 43$ & 0.7421 & 0.5302 & & \\
\hline Adjuvant therapy & In-completion & 1.9714 & 0.0990 & & \\
\hline \multicolumn{6}{|l|}{$<$ Pathological factor $>$} \\
\hline Tumor size & $\geqq 20$ & 2.8865 & 0.1536 & & \\
\hline T & T3 & 1.4130 & 0.5319 & & \\
\hline Differentiation & Moderate or poor & 1.6448 & 0.2581 & & \\
\hline Lymph node metastasis & $\mathrm{N} 2$ or N1 & 3.5747 & 0.0080 & 4.7976 & 0.0117 \\
\hline ly & 2 or 3 & 1.3131 & 0.59647 & & \\
\hline$v$ & 2 or 3 & 1.7984 & 0.1657 & & \\
\hline ne & 2 or 3 & 1.4089 & 0.4079 & & \\
\hline PV & 1 & 5.6948 & 0.0001 & 2.6003 & 0.1012 \\
\hline Surgical margins & R1 & 12.1127 & 0.0042 & 12.6514 & 0.0295 \\
\hline Evans & I or Ila & 2.1983 & 0.1255 & & \\
\hline
\end{tabular}

BR-A, borderline resectable with arterial invasion; BR-PV, borderline resectable with portal vein invasion; NACRT, neoadjuvant chemoradiotherapy; CEA, carcinoembryonic antigen; CA19-9, carbohydrate antigen 19-9; FDG-PET, fluorodeoxyglucose positron emission tomography; SUVmax, maximum standardized uptake value; NLR, neutrophil/lymphocyte ratio; Alb, albumin; GPS, Glasgow Prognostic Score; mGPS, modified Glasgow Prognostic Score; CRP, C-reactive protein; PV, portal vein 

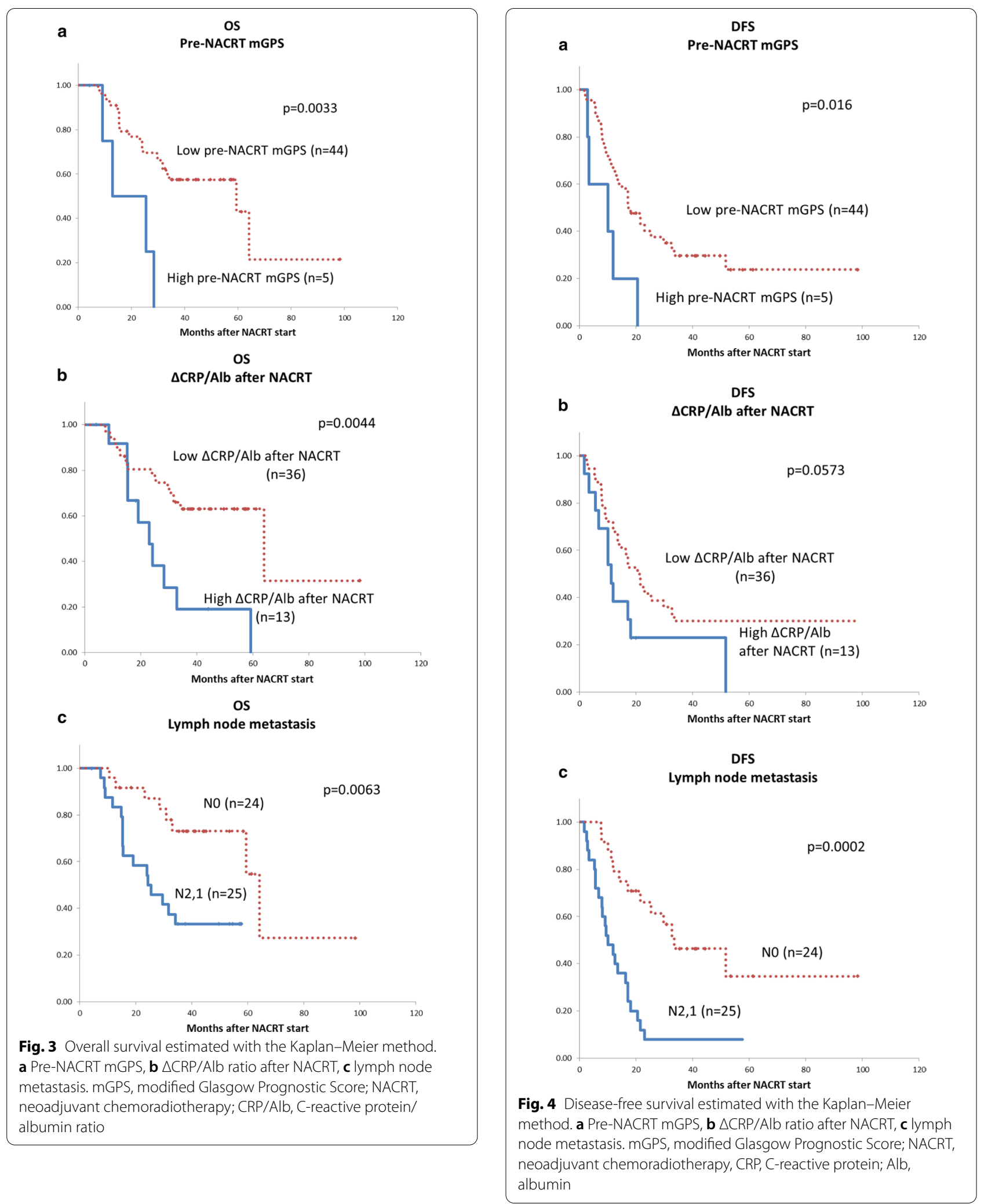
Table 3 Characteristics of patients in the groups with high $\Delta$ CRP/Alb ratio $(\geqq 0.077)$ and the group with low $\Delta$ CRP/Alb ratio $(<0.077)$

\begin{tabular}{|c|c|c|c|}
\hline & High $\Delta$ CRP/Alb group $(n=13)$ & Low $\Delta$ CRP/Alb group $(n=36)$ & $p$ value \\
\hline Age (years) & 74 & 68 & 0.1663 \\
\hline Sex: female & $6(46.2)$ & $16(44.4)$ & 0.916 \\
\hline Tumor size (mm) & 30 & 20 & 0.076 \\
\hline \multicolumn{4}{|l|}{$<$ Pre-NACRT $>$} \\
\hline CEA (ng/mL) & 5.5 & 3.9 & 0.1235 \\
\hline CA19-9 (U/mL) & 537 & 214 & 0.1889 \\
\hline FDG-PET (SUVmax) & 7.8 & 7.0 & 0.5926 \\
\hline Resectability: BR & $2(15.4)$ & $6(16.7)$ & 0.9155 \\
\hline BMI & 23.5 & 22.1 & 0.1129 \\
\hline CRP (mg/dL) & 0.41 & 0.16 & 0.0698 \\
\hline Alb (g/dL) & 3.8 & 3.9 & 0.7941 \\
\hline NLR & 2.51 & 2.00 & 0.0852 \\
\hline GPS 2 or 1 & $4(30.8)$ & $10(27.8)$ & 0.8395 \\
\hline mGPS 2 or 1 & $2(15.4)$ & $3(8.3)$ & 0.4761 \\
\hline \multicolumn{4}{|l|}{$<$ Post-NACRT> } \\
\hline CEA (ng/mL) & 6.5 & 3.5 & 0.1333 \\
\hline CA19-9 (U/mL) & 455 & 107 & 0.0728 \\
\hline$\Delta$ Weight loss (kg) & 1.55 & 0.48 & 0.0314 \\
\hline$C R P(m g / d L)$ & 1.32 & 0.08 & $<0.0001$ \\
\hline$A / b(g / d L)$ & 3.2 & 3.9 & 0.0015 \\
\hline NLR & 3.20 & 3.34 & 0.9639 \\
\hline GPS 2 or 1 & $11(84.6)$ & $10(27.8)$ & 0.0004 \\
\hline mGPS 2 or 1 & $10(76.9)$ & $0(0)$ & $<0.0001$ \\
\hline \multicolumn{4}{|l|}{$<$ Perioperative and postoperative factor $>$} \\
\hline Intraoperative bleeding (mL) & 1764 & 1005 & 0.0666 \\
\hline Complication after surgery ${ }^{a}$ & $2(15.4)$ & $10(27.8)$ & 0.3780 \\
\hline Postoperative hospital stays (days) & 29 & 25 & 0.5183 \\
\hline T3 & $2(15.4)$ & $5(13.9)$ & 0.8960 \\
\hline $\mathrm{N} 2$ or N1 & $7(53.8)$ & $18(50.0)$ & 0.8139 \\
\hline PVI & $5(38.5)$ & $10(27.8)$ & 0.4783 \\
\hline Surgical margin: R1 & $4(30.8)$ & $10(27.8)$ & 0.8395 \\
\hline Evans I or lla & $11(84.6)$ & $22(61.1)$ & 0.1252 \\
\hline Uncompleted adjuvant chemotherapy & $7(53.9)$ & $15(41.7)$ & 0.4539 \\
\hline
\end{tabular}

Data are median or $\mathrm{n}(\%)$

CRP, C-reactive protein; Alb, albumin; NACRT, neoadjuvant chemoradiotherapy; CEA, carcinoembryonic antigen; CA19-9, carbohydrate antigen 19-9; FDG-PET, fluorodeoxyglucose positron emission tomography; SUVmax, maximum standardized uptake value; borderline resectable; BMI, body mass index; NLR, neutrophil/ lymphocyte ratio; GPS, Glasgow Prognostic Score; mGPS, modified Glasgow Prognostic Score; PVI, portal vein invasion

a Complication after surgery: Clavien-Dindo $\geqq I l l a$

Although PDAC remains a highly lethal disease, neoadjuvant therapy has been considered to provide a new and effective treatment strategy for PDAC. It improves treatment outcome in resectable and borderline resectable cases and provides a survival benefit in patients with advanced stages of PDAC $[5,7,30]$. Because neoadjuvant therapy for resectable or borderline resectable PDAC is not yet a standard treatment, NACRT has been the subject of clinical trials at high-volume centers in Japan. Because of this, there are few studies that demonstrate the value of inflammatory nutritional prognostic scores in the patients undergoing NACRT for resectable or borderline resectable PDAC. This is the first report showing that increased CRP/Alb ratio after NACRT can be a valuable prognostic factor. Our previous study presented that neoadjuvant S-1 with concurrent hypofractionated radiotherapy is tolerable and appears promising for patients with resectable and borderline resectable PDAC [23]. Recently, a randomized phase II/III trial of neoadjuvant chemotherapy with gemcitabine and S-1 versus 
upfront surgery for resectable pancreatic cancer (Prep02/JSAP05) (clinical trial information: UMIN000009634) demonstrated that neoadjuvant chemotherapy contributed to improved treatment outcome and prognosis for resectable PDAC compared with upfront surgery [31]. Based on the efficacy of neoadjuvant chemotherapy in the planned resection of patients with PDAC, as determined by the trial, neoadjuvant therapy could become one of the standard established therapies for resectable or borderline resectable PDAC.

This study demonstrates that high pre-NACRT mGPS and increased CRP/Alb ratio after NACRT are independent prognostic factors for patients with resectable or borderline resectable PDAC undergoing NACRT following pancreatic resection, like lymph node metastasis and cancer remnant. Both OS and DFS estimated with the Kaplan-Meier method are poor in the patient group with high pre-NACRT mGPS. According to the patient group with increased CRP/Alb ratio after NACRT, OS was poor. Additionally, DFS was also inferior although the $p$ value did not reach statistical difference. Several papers have demonstrated that some preoperative inflammatory nutritional scores could be utilized as prognosis predictors $[15,20,21]$. Additionally, a study reported that an increase in NLR postoperative course leads to poor prognosis [32]. Furthermore, from the results of this study, a change in the degree of inflammatory nutritional scores after NACRT could also be valuable predictive markers. To our knowledge, there are only a few reports that focused on the relationship between NACRT for PDAC and change in inflammatory nutritional scores. Differences in race may affect the findings in the present study, as $\mathrm{S}-1$ is used as sensitizer in the protocol. Further examinations focusing on changes in inflammatory nutritional scores are required, because treatment protocols for NACRT differ among institutions.

To assess the cause of increased CRP/Alb ratio after NACRT, all the patients included in this study were divided into two groups according to $\triangle \mathrm{CRP} / \mathrm{Alb}$ ratio after NACRT: the group with high $\triangle$ CRP/Alb $(\geqq 0.077)$ and the group with low $-\triangle \mathrm{CRP} / \mathrm{Alb}$ ratio $(<0.077)$. The group with high $\triangle \mathrm{CRP} / \mathrm{Alb}$ after NACRT not only had higher post-NACRT CRP level but also lower postNACRT Alb level and lost more body weight after NACRT than the group with low- $\triangle C R P / A l b$. Previous studies reported that tumor malignant behavior and progression are associated with impaired Alb synthesis and malnutrition. However, tumor characteristics, including tumor size, tumor marker and lymph node metastasis, were not significantly different between the two groups, suggesting that low Alb levels and malnutrition are independently related to poor prognosis. Meanwhile, some papers have described sarcopenia or loss of skeletal muscle volume as adverse prognostic factors for pancreatic cancer [33-35]. Based on the reason for the high post-NACRT CRP level in the group with high $-\triangle C R P /$ Alb, the patients could be more prone to development of inflammation due to carcinogenesis and/or NACRT.

Some reported that predictive prognostic factors in PDAC, including tumor size, tumor marker, lymph node metastasis, differentiation, and molecular behavior, could not be intervened enough for the improvement of prognosis. Tumor behavior and pathological examinations are difficult to access before surgical resection. Meanwhile, assessment of immune nutritional status through CRP/ Alb ratio and mGPS are available from the first diagnosis up to the postoperative period. Most important, malnutrition and sarcopenia can be modified with appropriate intervention. Patients who undergo NACRT, especially those with low albumin level or with low body weight, should be given advance nutritional support because they have enough time to improve their albumin level and/or body weight before surgery.

Several limitations of the study should be mentioned. First, the number of patients in the study may not be sufficient to draw firm conclusions based on statistical analysis. Since the use of NACRT has not been standardized widely to date, collecting enough patients treated using the same protocols is currently difficult. The second limitation is that the inflammation and nutritional scores may be affected by progression of TNM stage due to the heterogeneous patient population in the study. We hope that the proposed consequences of the study will be verified by future large-scale research with subgroup analysis of patients at an identical stage.

\section{Conclusion}

Inflammatory nutritional prognostic scores could provide additional prognostic value even in patients with PDAC treated by NACRT. Further studies are needed in order to consider the possibility that advanced nutrition support during NACRT might be able to contribute to improving the prognosis.

\section{Supplementary Information}

The online version contains supplementary material available at https://doi. org/10.1186/s12876-020-01566-8.

Additional file 1: Table 1. Inflammatory nutritional prognostic scoring system.

\section{Abbreviations}

PDAC: Pancreatic ductal adenocarcinoma; NACRT: Neoadjuvant chemoradiotherapy; NLR: Neutrophil/lymphocyte ratio; GPS: Glasgow prognostic score; mGPS: Modified Glasgow prognostic score; CRP/Alb ratio: C-reactive protein/ albumin ratio; R: Resectable; BR: Borderline resectable; NCCN: The National Comprehensive Cancer Network; PD: Pancreaticoduodenectomy; DP: Distal 
pancreatectomy; RAMPS: Radical antegrade modular pancreatosplenectomy; OS: Overall survival; DFS: Disease-free-survival; BR-PV: Borderline resectable with portal vein invasion; BR-A: Borderline resectable with arterial invasion; CEA: Carcinoembryonic antigen; CA19-9:: Carbohydrate antigen 19-9; FDGPET: Fluorodeoxyglucose positron emission tomography; SUV max: Maximum standardized uptake value; BMI: Body mass index; PVI: Portal vein invasion.

\section{Acknowledgements}

The authors thank Drs. Reiji Haba and Kyuichi Kadota (Department of Diagnostic Pathology, Kagawa University Hospital) for their assistance in histopathological diagnosis.

\section{Authors' contributions}

$\mathrm{MO}, \mathrm{KO}$, and $\mathrm{YS}$ contributed to the study conception and design. $\mathrm{HS}$ and $\mathrm{YA}$ performed data collection. HK and TM diagnosed PDAC with endoscopic ultrasound-guided fine-needle aspiration. ST and TS performed radiotherapy. All authors read and approved the final manuscript.

\section{Funding}

This work did not receive any funding.

\section{Availability of data and materials}

The datasets generated and/or analyzed in the present study are available from the corresponding author on reasonable request.

\section{Ethics approval and consent to participate}

The study was conducted in compliance with the International Conference on Harmonization guidelines for Good Clinical Practice (E6) and the 2013 Declaration of Helsinki. All patients provided written informed consent before inclusion in the study, which was approved by the institutional review board of Kagawa University (Approval No 2009-14).

\section{Consent for publication}

Not applicable.

\section{Competing interests}

The authors declare no conflicts of interest associated with this manuscript.

\section{Author details}

1 Department of Gastroenterological Surgery, Faculty of Medicine, Kagawa University, 1750-1, Ikenobe, Miki-cho, Kita-gun, Kagawa 761-0793, Japan. ${ }^{2}$ Departments of Gastroenterology and Neurology, Faculty of Medicine, Kagawa University, 1750-1, Ikenobe, Miki-cho, Kita-gun, Kagawa 761-0793, Japan. ${ }^{3}$ Radiation Oncology, Faculty of Medicine, Kagawa University, 1750-1, Ikenobe, Miki-cho, Kita-gun, Kagawa 761-0793, Japan.

\section{Received: 7 September 2020 Accepted: 1 December 2020}

Published online: 14 December 2020

\section{References}

1. Siegel RL, Miller KD, Jemal A. Cancer statistics, 2017. CA. 2017:67:7-30.

2. Vincent A, Herman J, Schulick R, Hruban RH, Goggins M. Pancreatic cancer. Lancet. 2011:378:607-20.

3. Willett CG, Lewandrowski K, Warshaw AL, Efird J, Compton CC. Resection margins in carcinoma of the head of the pancreas. Implic Radiat Ther Ann Surg. 1993;217:144-8.

4. Labori KJ, Katz MH, Tzeng CW, Bjornbeth BA, Cvancarova M, Edwin B, et al. Impact of early disease progression and surgical complications on adjuvant chemotherapy completion rates and survival in patients undergoing the surgery first approach for resectable pancreatic ductal adenocarcinoma-a population-based cohort study. Acta Oncol. 2016;55:265-77.

5. Evans DB, Pisters PW, Lee JE, Bold RJ, Charnsangavej C, Janjan NA, et al. Preoperative chemoradiation strategies for localized adenocarcinoma of the pancreas. J Hepatobiliary Pancreat Surg. 1998;5:242-50.

6. Uesaka K, Boku N, Fukutomi A, Okamura Y, Konishi M, Matsumoto I, et al. Adjuvant chemotherapy of S-1 versus gemcitabine for resected pancreatic cancer: a phase 3, open-label, randomised, non-inferiority trial (JASPAC 01). Lancet. 2016;388:248-57.
7. Gillen S, Schuster T, Meyer Zum Buschenfelde C, Friess H, Kleeff J. Preoperative/neoadjuvant therapy in pancreatic cancer: a systematic review and meta-analysis of response and resection percentages. PLoS Med. 2010;7:e1000267.

8. Artinyan A, Anaya DA, McKenzie S, Ellenhorn JD, Kim J. Neoadjuvant therapy is associated with improved survival in resectable pancreatic adenocarcinoma. Cancer. 2011;117:2044-9.

9. Cloyd JM, Crane CH, Koay EJ, Das P, Krishnan S, Prakash L, et al. Impact of hypofractionated and standard fractionated chemoradiation before pancreatoduodenectomy for pancreatic ductal adenocarcinoma. Cancer. 2016;122:2671-9.

10. Byun Y, Han Y, Kang JS, Choi YJ, Kim H, Kwon W, et al. Role of surgical resection in the era of FOLFIRINOX for advanced pancreatic cancer. J Hepatob Pancreat Sci. 2019:26:416-25.

11. Sandhu V, Labori KJ, Borgida A, Lungu I, Bartlett J, Hafezi-Bakhtiari S, et al. Meta-analysis of 1,200 transcriptomic profiles identifies a prognostic model for pancreatic ductal adenocarcinoma. JCO Clin Cancer Inform. 2019;3:1-16.

12. Slidell MB, Chang DC, Cameron JL, Wolfgang C, Herman JM, Schulick RD, et al. Impact of total lymph node count and lymph node ratio on staging and survival after pancreatectomy for pancreatic adenocarcinoma: a large, population-based analysis. Ann Surg Oncol. 2008;15:165-74.

13. Luttges J, Schemm S, Vogel I, Hedderich J, Kremer B, Kloppel G. The grade of pancreatic ductal carcinoma is an independent prognostic factor and is superior to the immunohistochemical assessment of proliferation. J Pathol. 2000;191:154-61.

14. Tas F, Karabulut S, Ciftci R, Sen F, Sakar B, Disci R, et al. Serum levels of LDH, CEA, and CA19-9 have prognostic roles on survival in patients with metastatic pancreatic cancer receiving gemcitabine-based chemotherapy. Cancer Chemother Pharmacol. 2014;73:1163-71.

15. Ahmad J, Grimes N, Farid S, Morris-Stiff G. Inflammatory response related scoring systems in assessing the prognosis of patients with pancreatic ductal adenocarcinoma: a systematic review. Hepatobiliary Pancreat Dis Int. 2014;13:474-81.

16. McMillan DC. The systemic inflammation-based Glasgow prognostic score: a decade of experience in patients with cancer. Cancer Treatment Rev. 2013;39:534-40.

17. Mei Z, Shi L, Wang B, Yang J, Xiao Z, Du P, et al. Prognostic role of pretreatment blood neutrophil-to-lymphocyte ratio in advanced cancer survivors: a systematic review and meta-analysis of 66 cohort studies. Cancer Treatment Rev. 2017;58:1-13.

18. Forrest LM, McMillan DC, MCArdle CS, Angerson WJ, Dunlop DJ. Comparison of an inflammation-based prognostic score (GPS) with performance status (ECOG) in patients receiving platinum-based chemotherapy for inoperable non-small-cell lung cancer. Br J Cancer. 2004;90:1704-6.

19. McMillan DC, Crozier JE, Canna K, Angerson WJ, McArdle CS. Evaluation of an inflammation-based prognostic score (GPS) in patients undergoing resection for colon and rectal cancer. Int J Colorectal Dis. 2007;22:881-6.

20. Yamada S, Fujii T, Yabusaki N, Murotani K, Iwata N, Kanda M, et al. Clinical implication of inflammation-based prognostic score in pancreatic cancer: Glasgow prognostic score is the most reliable parameter. Medicine. 2016;95:e3582.

21. Liu Z, Jin K, Guo M, Long J, Liu L, Liu C, et al. Prognostic value of the crp/ alb ratio, a novel inflammation-based score in pancreatic cancer. Ann Surg Oncol. 2017:24:561-8.

22. Chen Y, Chen K, Xiao X, Nie Y, Qu S, Gong C, et al. Pretreatment neutrophil-to-lymphocyte ratio is correlated with response to neoadjuvant chemotherapy as an independent prognostic indicator in breast cancer patients: a retrospective study. BMC Cancer. 2016;16:320.

23. Okano K, Suto H, Oshima M, Maeda E, Yamamoto N, Kakinoki K, et al. A prospective phase ii trial of neoadjuvant s-1 with concurrent hypofractionated radiotherapy in patients with resectable and borderline resectable pancreatic ductal adenocarcinoma. Ann Surg Oncol. 2017:24:2777-84.

24. National Comprehensive Cancer Network. Pancreatic adenocarcinoma. Available at: http://www.nccnorg/professionals/physician_gls/pdf/pancr eaticpdf (2016)

25. Oshima M, Okano K, Muraki S, Haba R, Maeba T, Suzuki Y, et al. Immunohistochemically detected expression of 3 major genes (CDKN2A) p16, TP53, and SMAD4/DP(4) strongly predicts survival in patients with resectable pancreatic cancer. Ann Surg. 2013;258:336-46. 
26. Stotz M, Gerger A, Eisner F, Szkandera J, Loibner H, Ress AL, et al. Increased neutrophil-lymphocyte ratio is a poor prognostic factor in patients with primary operable and inoperable pancreatic cancer. Br J Cancer. 2013;109:416-21.

27. Bhatti I, Peacock O, Lloyd G, Larvin M, Hall RI. Preoperative hematologic markers as independent predictors of prognosis in resected pancreatic ductal adenocarcinoma: neutrophil-lymphocyte versus platelet-lymphocyte ratio. Am J Surg. 2010;200:197-203.

28. La Torre M, Ziparo V, Nigri G, Cavallini M, Balducci G, Ramacciato G. Malnutrition and pancreatic surgery: prevalence and outcomes. J Surg Oncol. 2013;107:702-8.

29. Argiles JM. Cancer-associated malnutrition. Eur J Oncol Nurs. 2005;9(Suppl 2):S39-50.

30. Verma V, Li J, Lin C. Neoadjuvant therapy for pancreatic cancer: systematic review of postoperative morbidity, mortality, and complications. Am J Clin Oncol. 2016;39:302-13.

31. Motoi F, Kosuge T, Ueno H, Yamaue H, Satoi S, Sho M, et al. Randomized phase II/III trial of neoadjuvant chemotherapy with gemcitabine and S-1 versus upfront surgery for resectable pancreatic cancer (Prep-02/JSAP05). Jpn J Clin Oncol. 2019;49:190-4.
32. Chen Y, Yan H, Wang Y, Shi Y, Dai G. Significance of baseline and change in neutrophil-to-lymphocyte ratio in predicting prognosis: A retrospective analysis in advanced pancreatic ductal adenocarcinoma. Sci Rep. 2017;7:753.

33. Tan BH, Birdsell LA, Martin L, Baracos VE, Fearon KC. Sarcopenia in an overweight or obese patient is an adverse prognostic factor in pancreatic cancer. Clin Cancer Res. 2009;15:6973-9.

34. Peng P, Hyder O, Firoozmand A, Kneuertz P, Schulick RD, Huang D, et al. Impact of sarcopenia on outcomes following resection of pancreatic adenocarcinoma. J Gastrointest Surg. 2012;16:1478-86.

35. Okumura S, Kaido T, Hamaguchi Y, Fujimoto Y, Masui T, Mizumoto M, et al. Impact of preoperative quality as well as quantity of skeletal muscle on survival after resection of pancreatic cancer. Surgery. 2015;157:1088-98.

\section{Publisher's Note}

Springer Nature remains neutral with regard to jurisdictional claims in published maps and institutional affiliations.
Ready to submit your research? Choose BMC and benefit from:

- fast, convenient online submission

- thorough peer review by experienced researchers in your field

- rapid publication on acceptance

- support for research data, including large and complex data types

- gold Open Access which fosters wider collaboration and increased citations

- maximum visibility for your research: over $100 \mathrm{M}$ website views per year

At BMC, research is always in progress.

Learn more biomedcentral.com/submissions 